\title{
Metformin inhibits the proliferation of A549/CDDP cells by activating p38 mitogen-activated protein kinase
}

\author{
YAJUN WANG ${ }^{1}$, BIYUN LIN ${ }^{2}$, JUN WU $^{1}$, HAITAO ZHANG $^{2}$ and BIN WU ${ }^{1}$ \\ ${ }^{1}$ Institute of Respiratory Diseases, Affiliated Hospital of Guangdong Medical College; \\ ${ }^{2}$ Department of Biochemistry and Molecular Biology, Guangdong Medical College, \\ Zhanjiang, Guangdong 524023, P.R. China
}

Received November 19, 2013; Accepted May 23, 2014

DOI: $10.3892 / \mathrm{ol} .2014 .2270$

\begin{abstract}
Metformin (Met) has been widely used in hypoglycemic therapy, and it is also able to reduce the incidence of tumors and tumor-related mortality. The present study investigated whether Met could inhibit the proliferation of lung cancer cells and enhance the sensitivity of a cisplatin-resistant lung cancer A549/CDDP cell line to cisplatin. It was found that Met treatment inhibited the proliferation of different lung cancer cells. Met inhibited the cell cycle of the A549/CDDP cells and induced apoptosis. Upon Met treatment, the A549/CDDP cells were arrested at the $G_{1}$ phase. The apoptosis of the A549/CDDP cells was confirmed by the appearance of apoptotic bodies in cells stained with Hoechst 33258, and by the cleavage of $\mathrm{BH} 3$ interacting-domain death agonist and poly (ADP-ribose) polymerase. Furthermore, results showed that the phosphorylation level of p38 mitogen-activated protein kinase (MAPK) was increased after Met treatment. The p38 MAPK inhibitor, SB203580, significantly blocked Met-induced apoptosis in the A549/CDDP cells. It was further demonstrated that Met could enhance the sensitivity of the A549/CDDP cells to cisplatin. In summary, the present study identified Met as a drug sensitizer that could improve the treatment effect of cisplatin in cisplatin-resistant lung cancers.
\end{abstract}

\section{Introduction}

Lung cancer is one of the most common malignant tumors in the world. Clinical trials have shown that cisplatin-based chemotherapy can significantly improve the survival rate of

Correspondence to: Professor Jun $\mathrm{Wu}$ or Professor Bin $\mathrm{Wu}$, Institute of Respiratory Diseases, Affiliated Hospital of Guangdong Medical College, Guangdong Medical College, 57 Renmin Avenue South, Zhanjiang, Guangdong 524023, P.R. China E-mail:wujun0294@163.com

E-mail: wubin621011@126.com

Abbreviations: Met, metformin; MAPK, mitogen-activated protein kinase

Key words: metformin, cisplatin-resistant lung cancer cells, p38 MAPK inoperable non-small cell lung cancer patients (1), but resistance to cisplatin limits its wider use in clinical applications. At present, the overall five-year survival rate of lung cancer patients is $<15 \%$ (2). Cisplatin resistance is the main reason for the failure of cancer chemotherapy, which contributes to the difficulty in providing a lung cancer cure and a poor long-term survival rate. Therefore, finding an effective method or medicine to reverse cisplatin resistance is a reasonable strategy to solve the drug resistance problem in lung cancer. To this end, the study of the mechanism of cisplatin resistance in lung cancer is key. During chemotherapy, cisplatin takes effect through the induction of lung cancer cell apoptosis. The resistance to cisplatin-induced cell apoptosis is considered as one of the important mechanisms of drug resistance in lung cancer (3).

Metformin (Met) has been widely used in hypoglycemic therapy in patients with type 2 diabetes (4). It has been shown in clinical observations that Met could reduce the incidence of tumors and the mortality of cancer patients (5). In recent years, numerous studies have confirmed that Met has anti-cancer effects (5-10). However the mechanism with regard to how Met affects the proliferation of lung cancer is not clear. It is also unclear whether Met could increase the sensitivity of cancer cells to cisplatin treatment. The present study investigated the effect of Met on the proliferation of different lung cancer cells and its effect on the cisplatin resistance. The study also provides a discussion on the mechanism of Met action, and conducts a preliminary evaluation of Met as an anticancer drug.

\section{Materials and methods}

Cell culture. The human lung cancer A549, cisplatin-resistant lung cancer A549/CDDP, SPCA and H23 cell lines were kept in the Institute of Biochemistry and Molecular Biology in Guangdong Medical College (Zhanjiang, Guangdong, China). The cell lines were cultured in the Dulbecco's modified Eagle's medium (Gibco BRL, Carlsbad, CA, USA) supplemented with $10 \%$ fetal bovine serum (Sijiqing Laboratories,Hangzhou, Zhejiang, China), $100 \mu \mathrm{g} / \mathrm{ml}$ penicillin and $100 \mu \mathrm{g} / \mathrm{ml}$ streptomycin. The cells were incubated at $37^{\circ} \mathrm{C}$ in a humidified atmosphere with $5 \% \mathrm{CO}_{2}$.

Reagents. Met and MTT were purchased from Sigma-Aldrich (St. Louis, MO, USA). Rabbit and goat polyclonal antibodies 
against cytochrome $c$, BH3 interacting-domain death agonist (Bid), poly (ADP-ribose) polymerase (PARP), caspase-3, caspase-8, $\beta$-actin and SB203580 were purchased from Santa Cruz Biotechnology, Inc. (Santa Cruz, CA, USA). The antibodies against phosphorylated forms of p38 MAPK-Thr180/Tyr182 and against p38 MAPK were purchased from Cell Signaling Technology (Beverly, MA, USA).

Cell viability assay. The A549, A549/CDDP, SPCA and $\mathrm{H} 23$ cells were treated with different concentrations of Met $(0$, $0.5,1,2,4$ and $8 \mathrm{mmol} / \mathrm{l})$ for various times $(24,48$ and $72 \mathrm{~h})$ and the cell viability was determined by MTT assay, as previously described (11).

Analysis of cell apoptosis by fluorescence staining. The A549/CDDP cells were treated with $4 \mathrm{mM}$ Met for the different time periods at $37^{\circ} \mathrm{C}$. Priot to the cells being examined under a fluorescence microscope, they were incubated in Hoechst $33258(10 \mathrm{mg} / \mathrm{l})$ solution at $37^{\circ} \mathrm{C}$ for $20 \mathrm{~min}$. Apoptosis was evaluated by the uptake of Hoechst 33342 (12). The apoptotic index was determined by dividing the number of apoptotic nuclei by the number of total nuclei.

Analysis of the cell cycle by flow cytometry. The cells were collected by centrifugation at $1,000 \mathrm{x} \mathrm{g}$ following treatment with $4 \mathrm{mM}$ Met for the different time periods at $37^{\circ} \mathrm{C}$. The cells were then washed twice with phosphate-buffered saline (PBS) and fixed with ice-cold 70\% ethanol overnight. Prior to the flow cytometry analysis for cell cycle distribution, the fixed cells were washed once with PBS (Sigma-Aldrich) and incubated with $100 \mu \mathrm{g} / \mathrm{ml}$ propidium iodide (Sigma-Aldrich) plus $200 \mu \mathrm{g} / \mathrm{ml}$ RNase (Sigma-Aldrich).

Treatment with $p 38$ MAPK inhibitors. The ells were preincubated with the MAPK inhibitor, SB203580 $(10 \mu \mathrm{M})$, for $2 \mathrm{~h}$ and then treated with Met $(4 \mathrm{mM})$ for $24 \mathrm{~h}$. Cell viability was determined using an MTT assay and the protein levels were measured by western blotting.

Western blotting. The cells were lysed with cell lysis buffer (pH 8.0) containing $50 \mathrm{mM}$ Tris-HCl (Sigma-Aldrich), $150 \mathrm{mM} \mathrm{NaCl}$ (Guangzhou Chemical Reagent Factory, Guangzhou, China), $5 \mathrm{mM}$ EDTA (Guangzhou Chemical Reagent Factory), 1\% NP40 (Sigma-Aldrich), 0.05\% phenylmethanesulfonyl fluoride (Sigma-Aldrich), $2 \mu \mathrm{g} / \mathrm{ml}$ aprotinin (Sigma-Aldrich) and $2 \mu \mathrm{g} / \mathrm{ml}$ leupeptin (Sigma-Aldrich). The protein levels were determined by western blotting, as described previously (4).

Treatment of A549/CDDP cells with Met and cisplatin. The A549/CDDP cells were treated with the different concentrations of Met and cisplatin for $24 \mathrm{~h}$ at $37^{\circ} \mathrm{C}$. Cell viability was determined using an MTT assay.

Statistical analysis. Results are presented as the mean \pm standard deviation. Statistical analysis was performed using a one-way analysis of variance with a least significant difference test. $\mathrm{P}<0.05$ was considered to indicate a statistically significant difference.
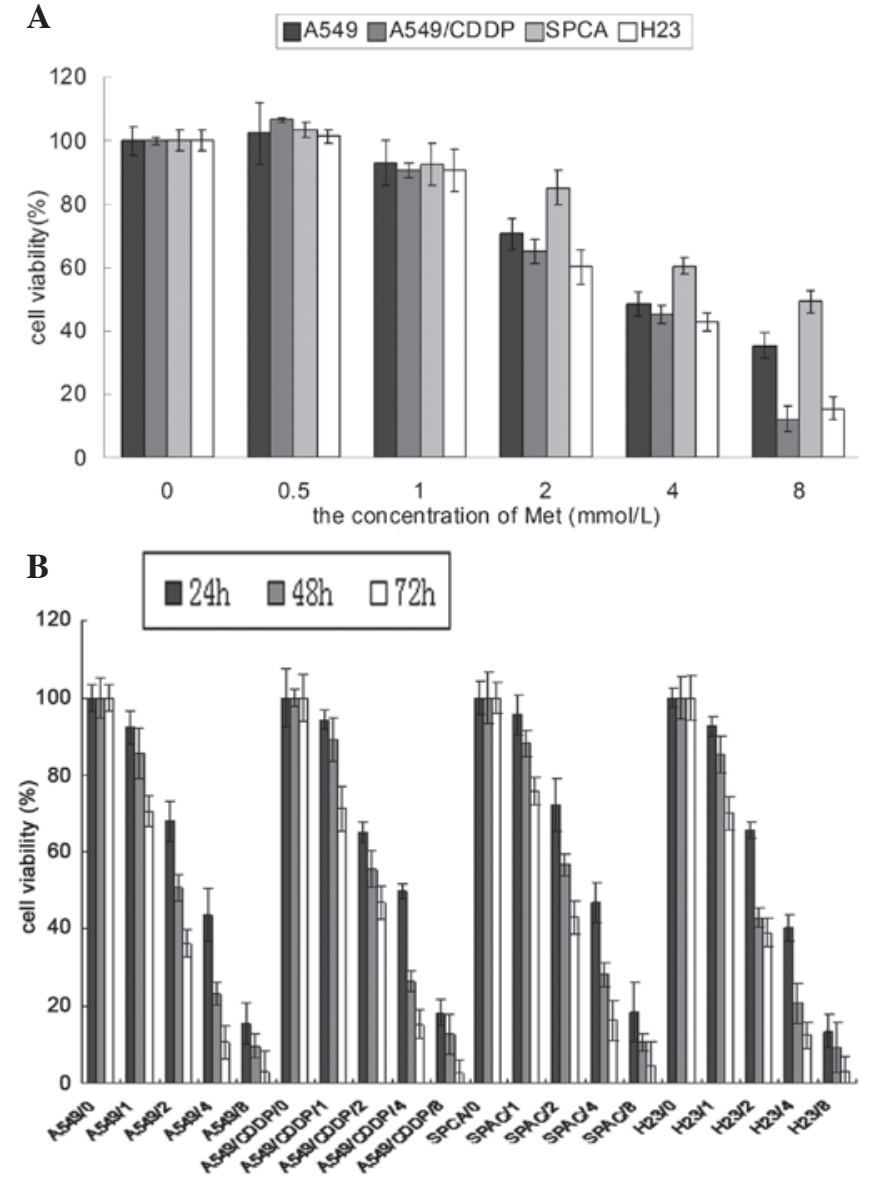

Figure 1. Effect of Met on the growth of the different lung cancer cell lines. (A) Different lung cancer cells were treated with varying concentrations of Met for $24 \mathrm{~h}$. The data are presented as the mean \pm standard deviation $(n=4)$. (B) Different lung cancer cells were treated with varying concentrations of Met for a range of times. The data are presented as the mean \pm standard deviation $(\mathrm{n}=4)$. Met, metformin.

\section{Results}

Effect of Met on the growth of different lung cancer cell lines. Fig. 1 showed that Met inhibited the proliferation of the lung cancer cells in a concentration- and time-dependent manner. The proliferation of the lung cancer cells was significantly inhibited by $24 \mathrm{~h}$ of Met treatment at concentrations of $2-8 \mathrm{mmol} / \mathrm{l}(\mathrm{P}<0.05)$. The survival of the lung cancer cells decreased significantly compared with the control group following $48 \mathrm{~h}$ of treatment with $1 \mathrm{mmol} / \mathrm{l}$ Met $(\mathrm{P}<0.05)$. It was also shown that the response of the A549/CDDP cells was similar to its parent cell line, A549.

Met inhibits the A549/CDDP cell cycle and induces apoptosis. The results showed that a large number of apoptotic cells appeared following 24-48 h of Met treatment (Fig. 2A). This was demonstrated by the apoptotic bodies in the cells stained with Hoechst 33258 (Fig. 2B). With Met treatment, the number of cells in the $G_{1}$ phase was increased, and the number of cells in the $\mathrm{S}$ phase and $\mathrm{G}_{2}$ phase was decreased (Fig. 2C).

Met induces the cleavage of Bid and PARP. The fragments of Bid and PARP increased with the increase in incubation 
A
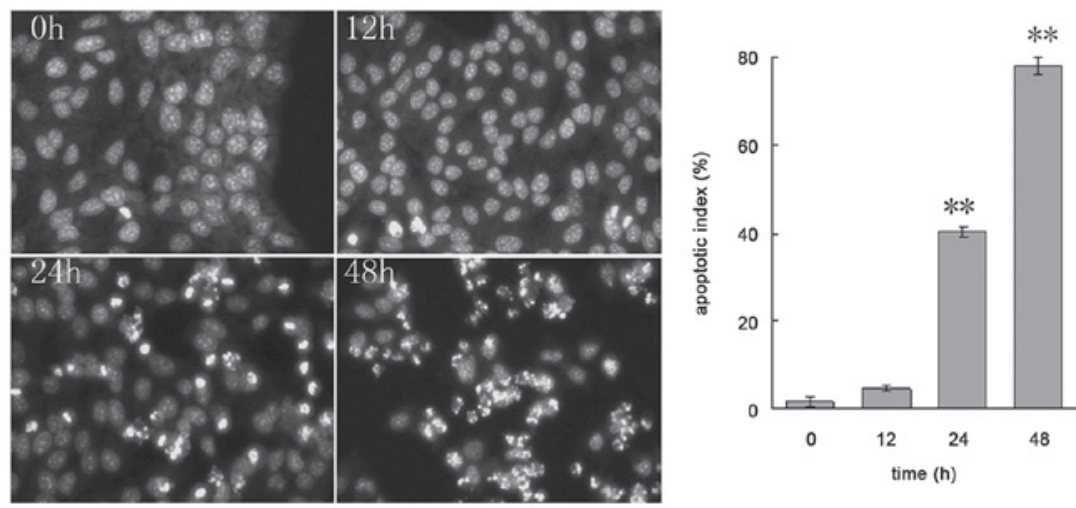

B
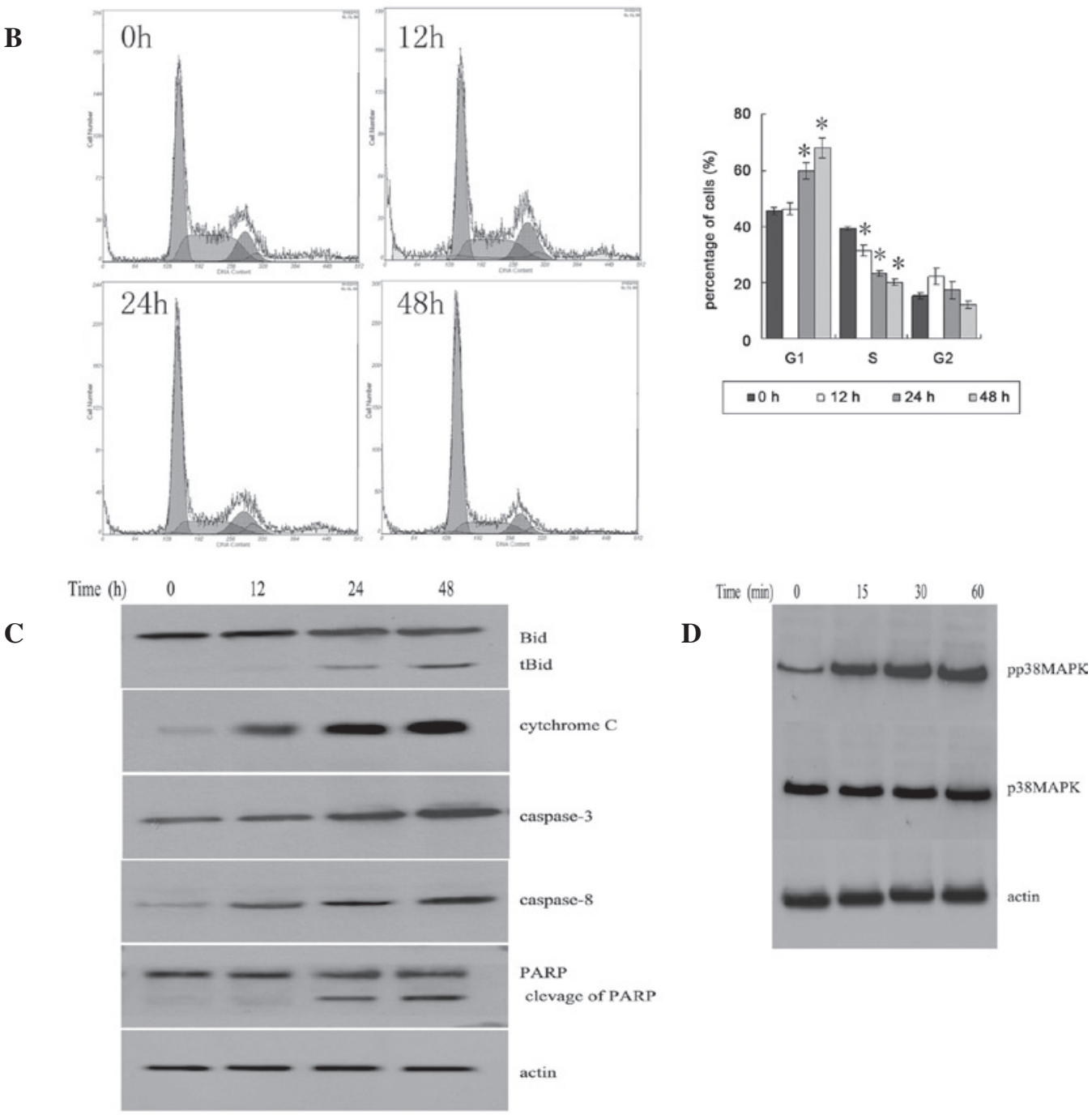

Figure 2. Met inhibits the A549/CDDP cell cycle and induces apoptosis. (A) Analysis of cell apoptosis by fluorescence staining. Images of the A549/CDDP cells following treatment with Met for different times. Nuclei were stained with Hoechst 33258 (magnification, x200). The apoptotic index was determined by calculating the percentage of the apoptotic nuclei as a portion of the total nuclei. Data are presented as the mean \pm standard deviation $(\mathrm{n}=4)$. ${ }^{*} \mathrm{P}<0.05$, ${ }^{* * *} \mathrm{P}<0.01 \mathrm{vs} .0 \mathrm{~h}$ group, respectively. (B) Flow cytometric analysis of cell cycle distribution after different periods of Met treatment. Data are presented as the mean \pm standard deviation (n=3). ${ }^{*} \mathrm{P}<0.05,{ }^{* * *} \mathrm{P}<0.01$ vs. 0 h group, respectively. (C) The expression levels of proteins as determined by western blotting. (D) The level of p38 MAPK as determined by western blotting. Met, metformin; MAPK, mitogen-activated protein kinase; PARP, poly (ADP-ribose) polymerase; Bid, BH3 interacting-domain death agonist.

time. The levels of caspase-3, caspase- 8 and cytochrome $c$ increased in the cytosol (Fig. 2C). It was shown that the phosphorylation level of p38 MAPK was increased following 15 min of Met treatment, indicating that Met could activate the p38 MAPK pathway (Fig. 2D).
Reduction of Met-induced cell apoptosis by inhibition of p38 MAPK activity. The A549/CDDP cells were preincubated with $10 \mu \mathrm{M}$ SB203580 (a p38 MAPK inhibitor) for $2 \mathrm{~h}$, and then treated with $4 \mathrm{mM}$ Met for $24 \mathrm{~h}$. The results showed that Met activated p38 MAPK and induced the apoptosis of the A549/CDDP 

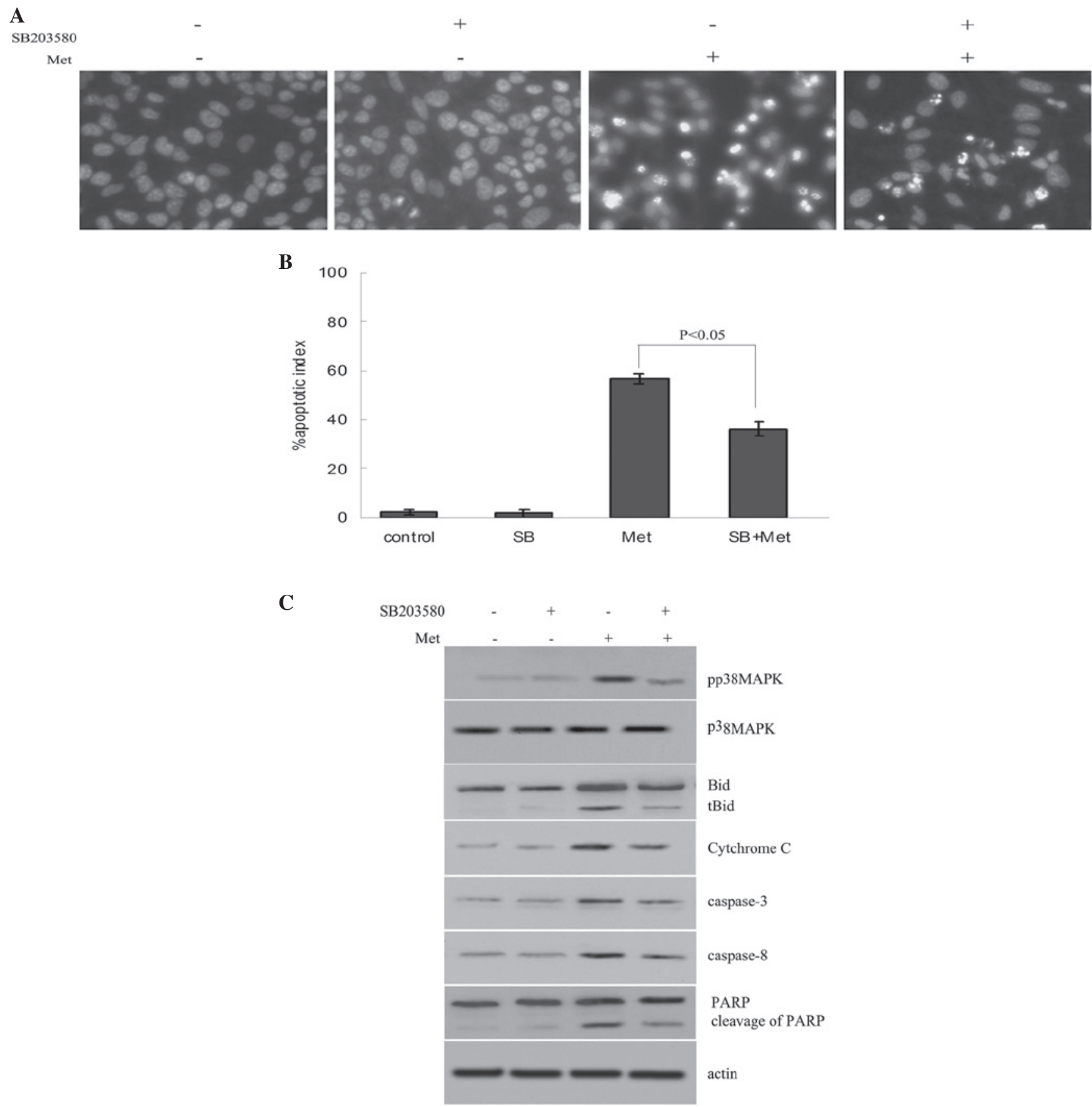

Figure 3. A549/CDDP cells were pre-incubated with $10 \mu \mathrm{M}$ SB203580 for $2 \mathrm{~h}$, followed by incubation with $4 \mathrm{mM}$ Met for 24 h. (A) Cells were stained with Hoechst 33258 (x200). (B) The apoptotic index was determined by calculating the percentage of the apoptotic nuclei as a proportion of the total nuclei. Data are presented as the mean \pm standard deviation $(n=4)$. (C) The expression levels of proteins as determined by western blotting. Met, metformin; MAPK, mitogen-activated protein kinase; PARP, poly (ADP-ribose) polymerase; Bid, BH3 interacting-domain death agonist; SB, SB203580 p38 MAPK inhibitor.

cells. Met-induced apoptosis was inhibited by SB203580 (Fig. 3A and B). The data also showed that SB203580 decreased the levels of Bid and PARP cleavage fragments, and the caspase-3, caspase-8 and cytosolic cytochrome $c$ levels (Fig. 3C).

Met enhances the sensitivity of A549/CDDP cells to cisplatin. Consistent with our previous study (12), the results showed that the $\mathrm{IC}_{50}$ values of cisplatin for the A549 cells and A549/CDDP cells were $2.36 \pm 0.10$ and $30.27 \pm 1.50 \mu \mathrm{mol} / 1$, respectively. The difference between the cisplatin-resistant and parent cells was 12.8-fold.
In this experiment, it was shown that the $\mathrm{IC}_{50}$ of cisplatin for the A549/CDDP cells was $32.18 \pm 1.15 \mu \mathrm{mol} /$ in the absence of Met, while the $\mathrm{IC}_{50}$ values decreased to $27.45 \pm 2.38,19.56 \pm 1.60$, $9.65 \pm 1.38,0.06 \pm 0.07$ and $0.00 \pm 0.05 \mu \mathrm{mol} / 1$ respectively in the presence of $0.5,1,2,4$ and $8 \mathrm{mmol} / 1 \mathrm{Met}(\mathrm{P}<0.05$ vs. control) (Fig. 4). Met evidently increased the cytotoxicity of cisplatin.

\section{Discussion}

Every year, 1,200,000 patients are diagnosed with lung cancer, and $25 \%$ of all cancer mortalities are patients with lung 


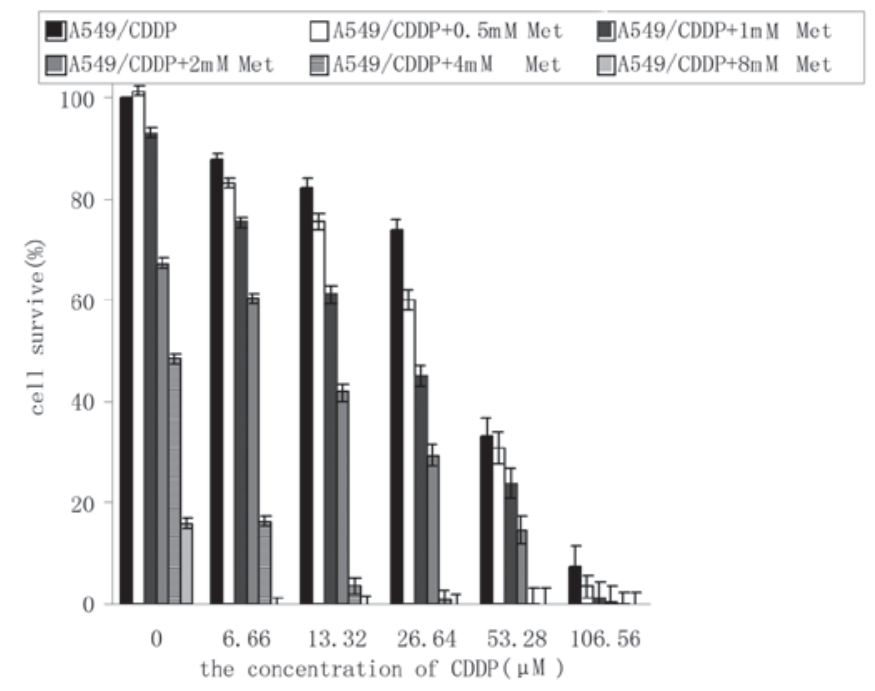

Figure 4. Met enhances the sensitivity of A549/CDDP cells to cisplatin (CDDP). The A549/CDDP cells were treated with different concentrations of Met and cisplatin for $24 \mathrm{~h}$. The data are presented as the mean \pm standard deviation $(n=4)$. Met, metformin.

cancer, representing a large threat to human health $(13,14)$. Chemotherapy and radiotherapy are the major methods for the treatment of patients with lung cancer. However, chemotherapy drugs have toxicity to normal tissue, and may cause serious side-effects in the clinic. Furthermore, the chemotherapy and radiotherapy drugs are expensive. Therefore it is of great import to search for effective antitumor drugs with a low toxicity and cost, which can enhance the effect of chemotherapy and improve the prognosis of lung cancer patients.

Met is an effective antihyperglycemic agent, which has been widely used in the treatment of patients with type 2 diabetes for decades. Certain studies have shown that Met not only inhibits breast cancer growth in HER-2/neu transgenic mice (6), but that it also inhibits pancreatic cancer induced by feeding of a high-fat diet in hamsters (7). Clinical data has also revealed that Met has anti-tumor activity. Evans et al (5) reported that the risk for tumorigenesis in type 2 diabetes patients administered Met treatment is $23 \%$ lower than those subjected to sulfonylurea treatment. Furthermore, after following 10,309 patients with newly diagnosed type 2 diabetes for approximately five years, Bowker et al (8) concluded that treatment with Met could be associated with lower mortality for malignancies in comparison with sulfonylureas in type 2 diabetes patients. Buzzai et al (9) demonstrated that Met has toxicity towards p53-mutated colorectal cancer cells. Ben Sahra et al (10) showed that Met inhibits the proliferation of prostate cancer cells, but not normal prostate epithelial cells. The present study data also showed that Met inhibited lung cancer cell growth and proliferation in a time- and concentration-dependent manner, which is consistent with previous results (5-9). In addition, the A549/CDDP cells were sensitive to Met. These findings suggest that Met may have a wide range of antitumor effects.

Cell proliferation is mainly affected by two factors, the cell cycle and apoptosis. Ben Sahra et al (10) showed that Met suppresses prostate cancer cell proliferation by arresting the cell cycle in the $G_{1}$ phase. However, it has no evident effect on cell apoptosis. However the present results demonstrated that Met not only induced A549/CDDP cell arrest at the $\mathrm{G}_{1}$ phase, but that it also promoted cell apoptosis.

Cisplatin is the most commonly used non-specific anticancer drug, which binds to tumor cell DNA and interferes with its function (15). The present study found no significant difference in the sensitivity to Met between the A549/CDDP cells and its parent cell line, A549. Furthermore, Met enhanced the sensitivity of the A549/CDDP cells to cisplatin when the cells were treated with Met and cisplatin together. The results suggest that Met may be used to enhance the cisplatin toxicity in cisplatin-resistant lung cancer cells and to improve the effectiveness of cisplatin-based chemotherapy in lung cancer patients.

Cisplatin has been used to treat tumors based on its ability to induce the apoptosis of tumor cells. One important mechanism of drug resistance in lung cancers is the tolerance of cancer cells to cisplatin-induced apoptosis. Apoptosis pathways include mainly the death receptor, mitochondrial and endoplasmic reticulum stress pathways. p38 MAPK and caspase family proteins play key roles in these apoptotic signal pathways.

The activation of p38 MAPK can induce apoptosis in various tumor cells. Jiang et al (16) reported that selenite activated p38 MAPK activity and promoted apoptosis in Jurkat cells. Inhibiting the p38 MAPK pathway can effectively reduce the release of cytochrome $c$ and inhibit Caspase-3 activation and PARP cleavage, resulting in the decrease of apoptosis cells (17). Mandal et al (18) showed that the p38 MAPK pathway is indispensable in the apoptosis of leukemia cells induced by Withaferin A. The p38 MAPK signaling pathway plays a key role in promoting the apoptosis of cells, a process associated with the activation of p53 and caspases, and the translocation of Bax (19,20). A study by Khan et al (21) demonstrated that the activation of the p38 MAPK pathway can induce the activation of caspases, and that the inhibition of p38 MAPK can reduce the expression levels of caspase activated protein. The study suggested that p38 MAPK is an upstream regulator of the caspase-dependent signal transduction pathway of apoptosis (21). The present study showed that Met induced the phosphorylation of the p38 MAPK protein and increased the expression of caspase- 8 and caspase-3, along with the cleavage of Bid and PARP, and cytochrome $c$ release in the cytoplasm. These results suggest that Met may promote the expression of caspase- 8 by activating p38 MAPK phosphorylation. Bid was cleaved by activated caspase- 8 to produce $\mathrm{tBid}$, which migrated to the mitochondrial membrane, resulting in the change of mitochondrial membrane permeability, cytochrome $c$ release, caspase-3 activation and cleavage of PARP to promote apoptosis. Inhibition of p38 MAPK significantly reduced the Met-induced apoptosis of the A549/CDDP cells. These results indicate that the p38 MAPK-caspase pathway may be involved in the regulation of A549/CDDP cell apoptosis induced by Met. Although the inhibition of the p38 MAPK signal pathway reduced the level of Met-induced A549/CDDP cell apoptosis, it did not completely inhibit the apoptosis of the A549/CDDP cells. This result suggests that other factors may participate in the regulation of A549/CDDP cell apoptosis induced by Met.

Results of the present study support the hypothesis that the proliferation of A549/CDDP cells can be inhibited by Met, 
which may be used as an adjuvant therapy to improve the clinical treatment effect of cisplatin in cisplatin-resistant lung cancers. Met is widely used as an insulin sensitizer and has shown few toxic side-effects in a number of years of clinical practice. Further studies are required to explore the other clinical applications of Met.

\section{Acknowledgements}

This project was supported by the Medical Scientific Research Foundation of Guangdong Province (A2011439), the Administration of Traditional Chinese Medicine of Guangdong Province (No.20111239) and the Startup Foundation for Doctors of The Affiliated Hospital of Guangdong Medical College (no. 10301B010014).

\section{References}

1. Winton T, Livingston R, Johnson D, Rigas J, Johnston M, Butts C, et al; National Cancer Institute of Canada Clinical Trials Group; National Cancer Institute of the United States Intergroup JBR.10 Trial Investigators: Vinorelbine plus cisplatin vs. observation in resected non-small-cell lung cancer. N Engl J Med 352: 2589-2597, 2005.

2. Risch A and Plass C: Lung cancer epigenetics and genetics. Int J Cancer 123: 1-7, 2008.

3. Stewart DJ: Mechanisms of resistance to cisplatin and carboplatin. Crit Rev Oncol Hematol 163: 12-31, 2007.

4. Gallwitz B and Bretzel RG: How do we continue treatment in patients with type 2 diabetes when therapeutic goals are not reached with oral antidiabetes agents and lifestyle? Incretin versus insulin treatment. Diabetes Care 36: S180-S189, 2013.

5. Evans JM, Donnelly LA, Emslie-Smith AM, Alessi DR and Morris AD: Metformin and reduced risk of cancer in diabetic patients. BMJ 330: 1304-1305, 2005.

6. Schneider MB, Matsuzaki H, Haorah J, Ulrich A, Standop J, Ding XZ, Adrian TE and Pour PM: Prevention of pancreatic cancer induction in hamsters by metformin. Gastroenterology 120 1263-1270, 2001.

7. Anisimov VN, Berstein LM, Egormin PA, Piskunova TS, Popovich IG, Zabezhinski MA,Kovalenko IG, Poroshina TE, Semenchenko AV, Provinciali M, Re F and Franceschi C: Effect of metformin on life span and on the development of spontaneousmammary tumors in HER-2/neu transgenic mice. Exp Gerontol 40: 685-693, 2005.

8. Bowker SL, Majumdar SR, Veugelers P and Johnson JA: Increased cancer-related mortality for patients with type 2 diabetes who use sulfonylureas or insulin. Diabetes Care 29: 254-258, 2006.
9. Buzzai M, Jones RG, Amaravadi RK, Lum JJ, DeBerardinis RJ, Zhao F, Viollet B and Thompson CB: Systemic treatment with the antidiabetic drug metformin selectively impairs p53-deficient tumor cell growth. Cancer Res 67: 6745-6752, 2007.

10. Ben Sahra I, Laurent K, Loubat A, Giorgetti-Peraldi S, Colosetti P, Auberger P, Tanti JF, Le Marchand-Brustel Y and Bost F: The antidiabetic drug metformin exerts an antitumoral effect in vitro and in vivo through a decrease of cyclin D1 level. Oncogene 27: 3576-3586, 2008.

11. Zhang HT, Wu J, Wen M, Su LJ and Luo H: Galangin induces apoptosis in hepatocellular carcinoma cells through the caspase 8/t-Bid mitochondrial pathway. J Asian Nat Prod Res 14: 626-633, 2012.

12. Wu J, Hu CP, Gu QH, Li YP and Song M: Trichostatin A sensitizes cisplatin-resistant A549 cells to apoptosis by up-regulating death-associated protein kinase. Acta Pharmacol Sin 31: 93-101, 2010.

13. Jemal A, Murray T, Ward E, Samuels A, Tiwari RC, Ghafoor A, Feuer EJ and Thun MJ: Cancer statistics, 2005. CA Cancer J Clin 55: 10-30, 2005.

14. Jemal A, Siegel R, Ward E, Murray T, Xu J and Thun MJ: Cancer statistics, 2007. CA Cancer J Clin 57: 43-66, 2007.

15. Florea AM and Büsselberg D: Cisplatin as an anti-tumor drug: cellular mechanisms of activity, drug resistance and induced side effects. Cancers (Basel) 15: 1351-1371, 2011.

16. Jiang Q, Li F, Shi K, Wu P, An J, Yang Y and Xu C: ATF4 activation by the p38MAPK-eIF4E axis mediates apoptosis and autophagy induced by selenite in Jurkat cells. FEBS Lett 587: 2420-2429, 2013

17. Neoh CA, Wang RY, Din ZH, Su JH, Chen YK, Tsai FJ, Weng SH and $\mathrm{Wu} \mathrm{YJ}$ : Induction of apoptosis by sinulariolide from soft coral through mitochondrial-related and $\mathrm{p} 38$ MAPK pathways on human bladder carcinoma cells. Mar Drugs 10: 2893-2911, 2012.

18. Mandal C, Dutta A, Mallick A, Chandra S, Misra L, Sangwan RS, Mandal C: Withaferin A induces apoptosis by activating p38 mitogen-activated protein kinase signaling cascade in leukemic cells of lymphoid and myeloid origin through mitochondrial death cascade. Apoptosis 13: 1450-1464, 2008.

19. Chang HL, Chen CY, Hsu YF, Kuo WS, Ou G, Chiu PT, Huang YH and Hsu MJ: Simvastatin induced HCT116 colorectal cancer cell apoptosis through p38MAPK-p53-survivin signaling cascade. Biochim Biophys Acta 1830: 4053-4064, 2013.

20. Yang X, Yao J, Luo Y, Han Y, Wang Z and Du L: P38 MAP kinase mediates apoptosis after genipin treatment in non-small-cell lung cancer H1299 cells via a mitochondrial apoptotic cascade. J Pharmacol Sci 121: 272-281, 2013.

21. Khan R, Khan AQ, Qamar W, Lateef A, Tahir M, Rehman MU, Ali F and Sultana S: Chrysin protects against cisplatin-induced colon toxicity via amelioration of oxidative stress and apoptosis: probable role of p38MAPK and p53. Toxicol Appl Pharmacol 258: 315-329, 2012. 